Article

\title{
Cohen-Macaulay and $\left(\mathrm{S}_{2}\right)$ Properties of the Second Power of Squarefree Monomial Ideals
}

\author{
Do Trong Hoang ${ }^{1,+}$, Giancarlo Rinaldo ${ }^{2,+}$ and Naoki Terai ${ }^{3, *,+}$ \\ 1 Institute of Mathematics, Vietnam Academy of Science and Technology, 18 Hoang Quoc Viet, \\ Hanoi 10307, Vietnam \\ 2 Department of Mathematics, University of Trento, via Sommarive, 14, 38123 Povo (Trento), Italy \\ 3 Faculty of Education, Saga University, Saga 840-8502, Japan \\ * Correspondence: terai@cc.saga-u.ac.jp \\ $\dagger$ These authors contributed equally to this work.
}

Received: 16 June 2019; Accepted: 30 July 2019; Published: 31 July 2019

\begin{abstract}
We show that Cohen-Macaulay and $\left(\mathrm{S}_{2}\right)$ properties are equivalent for the second power of an edge ideal. We give an example of a Gorenstein squarefree monomial ideal $I$ such that $S / I^{2}$ satisfies the Serre condition $\left(\mathrm{S}_{2}\right)$, but is not Cohen-Macaulay.
\end{abstract}

Keywords: Stanley-Reisner ideal; edge ideal; Cohen-Macaulay; $\left(\mathrm{S}_{2}\right)$ condition

\section{Introduction}

Let $K$ be a fixed field. Let $S=K\left[x_{1}, \ldots, x_{n}\right]$ be a polynomial ring with deg $x_{i}=1$ for all $i \in[n]=\{1,2, \ldots, n\}$. Let $I$ be a squarefree monomial ideal.

For a Stanley-Reisner ring $S / I$, the Cohen-Macaulay and $\left(S_{2}\right)$ properties are different in general. For instance, consider the Stanley-Reisner ring of a non-Cohen-Macaulay manifold, e.g., a torus, which satisfies the $\left(S_{2}\right)$ condition. However, for some special classes of such rings, they are known to be equivalent. The quotient ring of the edge ideal of a very well-covered graph (see [1]) and a Stanley-Reisner ring with "large" multiplicity (see [2] for the precise statement) are such examples. What about the powers of squarefree monomial ideals?

As for the third and larger powers, the following is proven in [3]:

Theorem 1. Let I be a squarefree monomial ideal. Then, the following conditions are equivalent for a fixed integer $m \geq 3$ :

1. $S / I$ is a complete intersection.

2. $S / I^{m}$ is Cohen-Macaulay.

3. $S / I^{m}$ satisfies the Serre condition $\left(S_{2}\right)$.

Then, what about the second power of a squarefree monomial ideal? This is the theme of this article. If the second power $I^{2}$ is Cohen-Macaulay, $I$ is not necessarily a complete intersection. Gorenstein ideals with height three give such examples.

In Section 3, we prove that the Cohen-Macaulay and $\left(\mathrm{S}_{2}\right)$ properties are equivalent for the second power of a squarefree monomial ideal generated in degree two:

Theorem 2. Let I be a squarefree monomial ideal generated in degree two. Then, the following conditions are equivalent:

1. $S / I^{2}$ is Cohen-Macaulay. 


\section{2. $S / I^{2}$ satisfies the Serre condition $\left(S_{2}\right)$.}

In Section 4, we first give an upper bound of the number of variables in terms of the dimension of $S / I$ when $I$ is a squarefree monomial ideal generated in degree two and $S / I^{2}$ has the Cohen-Macaulay (equivalently $\left(\mathrm{S}_{2}\right)$ ) property. Using a computer, we classify squarefree monomial ideals $I$ generated in degree two with $\operatorname{dim} S / I \leq 4$ such that $S / I^{2}$ have the Cohen-Macaulay (equivalently $\left(S_{2}\right)$ ) property. Since not many examples of squarefree monomial ideals $I$ generated in degree two such that $S / I^{2}$ are Cohen-Macaulay are known, new examples might be useful. See $[4,5]$ for the two- and three-dimensional cases, respectively, and $[6,7]$ for the higher dimensional case. See also $[6,8]$ for the fact that for a very well-covered graph $G$, the second power $I(G)^{2}$ is not Cohen-Macaulay if the edge ideal $I(G)$ of $G$ is not a complete intersection.

In Section 5, we give an example of a Gorenstein squarefree monomial ideal $I$ such that $S / I^{2}$ satisfies the Serre condition $\left(\mathrm{S}_{2}\right)$, but is not Cohen-Macaulay. Hence, the Cohen-Macaulay and $\left(\mathrm{S}_{2}\right)$ properties are different for the second power in general.

\section{Preliminaries}

\subsection{Stanley-Reisner Ideals}

We recall some notation on simplicial complexes and their Stanley-Reisner ideals. We refer the reader to [9-11] for the detailed information.

Set $V=[n]=\{1,2, \ldots, n\}$. A nonempty subset $\Delta$ of the power set $2^{V}$ of $V$ is called a simplicial complex on $V$ if the following two conditions are satisfied: (i) $\{v\} \in \Delta$ for all $v \in V$, and (ii) $F \in \Delta$, $H \subseteq F$ imply $H \in \Delta$. An element $F \in \Delta$ is called a face of $\Delta$. The dimension of $F$, denoted by $\operatorname{dim} F$, is defined by $\operatorname{dim} F=|F|-1$. The dimension of $\Delta$ is defined by $\operatorname{dim} \Delta=\max \{\operatorname{dim} F: F \in \Delta\}$. We call a maximal face of $\Delta$ a facet of $\Delta$. Let $\mathcal{F}(\Delta)$ denote the set of all facets of $\Delta$. We call $\Delta$ pure if all its facets have the same dimension. We call $\Delta$ connected if for any pair $(p, q), p \neq q$, of vertices of $\Delta$, there is a chain $p=p_{0}, p_{1}, p_{2}, \ldots, p_{k}=q$ of vertices of $\Delta$ such that $\left\{p_{i-1}, p_{i}\right\} \in \Delta$ for $i=1,2, \ldots, k$.

The Stanley-Reisner ideal $I_{\Delta}$ of $\Delta$ is defined by:

$$
I_{\Delta}=\left(x_{i_{1}} x_{i_{2}} \cdots x_{i_{p}}: 1 \leq i_{1}<\cdots<i_{p} \leq n,\left\{x_{i_{1}}, \ldots, x_{i_{p}}\right\} \notin \Delta\right) .
$$

The quotient ring $K[\Delta]=K\left[x_{1}, \ldots, x_{n}\right] / I_{\Delta}$ is called the Stanley-Reisner ring of $\Delta$.

We say that $\Delta$ is a Cohen-Macaulay (resp. Gorenstein) complex if $K[\Delta]$ is a Cohen-Macaulay (resp. Gorenstein) ring. A Gorenstein complex $\Delta$ is called Gorenstein* if $x_{i}$ divides some minimal monomial generator of $I_{\Delta}$ for each $i$.

For a face $F \in \Delta$, the link and star of $F$ are defined by:

$$
\begin{aligned}
& \operatorname{link}_{\Delta} F=\{H \in \Delta: H \cup F \in \Delta, H \cap F=\varnothing\}, \\
& \operatorname{star}_{\Delta} F=\{H \in \Delta: H \cup F \in \Delta\} .
\end{aligned}
$$

The Stanley-Reisner ideal $I_{\Delta}$ of $\Delta$ has the minimal prime decomposition:

$$
I_{\Delta}=\bigcap_{F \in \mathcal{F}(\Delta)} P_{F},
$$

where $P_{F}=(x \in[n] \backslash F)$ for each $F \in \mathcal{F}(\Delta)$. We call $I_{\Delta}$ unmixed if all $P_{F}$ have the same height for $F \in \mathcal{F}(\Delta)$. Note that $\Delta$ is pure if and only if $I_{\Delta}$ is unmixed. We define the $\ell^{\text {th }}$ symbolic power of $I_{\Delta}$ by:

$$
I_{\Delta}^{(\ell)}=\bigcap_{F \in \mathcal{F}(\Delta)} P_{F}^{\ell}
$$


For a Noetherian ring $A$, the following condition $\left(S_{i}\right)$ for $i=1,2, \ldots$ is called Serre's condition:

$$
\left(S_{i}\right) \text { depth } A_{P} \geq \min \{\text { height } P, i\} \text { for all } P \in \operatorname{Spec}(A) \text {. }
$$

See [12] for more information for Stanley-Reisner rings satisfying Serre's condition $\left(\mathrm{S}_{i}\right)$.

To introduce a characterization of the $\left(S_{2}\right)$ property for the second symbolic power of a Stanley-Reisner ideal, we first define the diameter of a simplicial complex. Let $\Delta$ be a connected simplicial complex. For $p, q$ being two vertices of $\Delta$, the distance between $p$ and $q$ is the minimal length $k$ of chains $p=p_{0}, p_{1}, p_{2}, \ldots, p_{k}=q$ of vertices of $\Delta$ such that $\left\{p_{i-1}, p_{i}\right\} \in \Delta$ for $i=1,2, \ldots, k$. The diameter, denoted by diam $\Delta$, is the maximal distance between two vertices in $\Delta$. We set $\operatorname{diam} \Delta=\infty$ if $\Delta$ is disconnected. The $\left(S_{2}\right)$ property of the second symbolic power of a Stanley-Reisner ideal is characterized as follows:

Theorem 3. ([7], Corollary 3.3) Let $\Delta$ be a pure simplicial complex. Then, the following conditions are equivalent:

1. $S / I_{\Delta}^{(2)}$ satisfies $\left(S_{2}\right)$.

2. $\operatorname{diam}\left(\operatorname{link}_{\Delta} F\right) \leq 2$ for any face $F \in \Delta$ with $\operatorname{dim} \operatorname{link}_{\Delta} F \geq 1$.

\subsection{Edge Ideals}

Let $G$ be a graph, which means a finite simple graph, which has no loops and multiple edges. We denote by $V(G)$ (resp. $E(G)$ ) the set of vertices (resp. edges) of $G$. We call $F \subseteq V(G)$ an independent set of $G$ if any $e \in E(G)$ is not contained in $F$. The independence complex $\Delta(G)$ of $G$ is defined by:

$$
\Delta(G)=\{F \subset V(G): e \not \subset F \text { for any } e \in E(G)\}
$$

which is a simplicial complex on the vertex set $V(G)$. We define $\alpha(G)$ by:

$$
\alpha(G)=\operatorname{dim} \Delta(G)+1
$$

We define the neighbor set $N_{G}(a)$ of a vertex $a$ of $G$ by:

$$
N_{G}(a)=\{b \in V: a b \in E(G)\} .
$$

Set $N_{G}[a]:=\{a\} \cup N_{G}(a)$, which is called the closed neighbor set of a vertex $a$ of $G$. For $S \subseteq V(G)$, we denote by $G \backslash S$ the induced subgraph on the vertex set $V(G) \backslash S$. Set $G_{S}:=G \backslash N_{G}[S]$, where $N_{G}[S]:=\cup_{x \in S} N_{G}[x]$. If $S \in \Delta(G)$, then:

$$
\operatorname{link}_{\Delta(G)}(S)=\Delta\left(G_{S}\right)
$$

See ([11], Lemma 7.4.3). For $a b \in E(G)$, set $G_{a b}:=G \backslash\left(N_{G}(a) \cup N_{G}(b)\right)$.

Set $V(G)=\{1, \ldots, n\}$. Then, the edge ideal of $G$, denoted by $I(G)$, is a squarefree monomial ideal of $S=K\left[x_{1}, \ldots, x_{n}\right]$ defined by:

$$
I(G)=\left(x_{i} x_{j}:\left\{x_{i}, x_{j}\right\} \in E(G)\right) .
$$

Note that $I(G)=I_{\Delta(G)}$. We call $G$ well-covered (or unmixed) if $I(G)$ is unmixed.

Theorem $4([13,14])$. Let $G$ be a graph. Then, the following conditions are equivalent:

1. $G$ is triangle-free.

2. $I(G)^{(2)}=I(G)^{2}$. 
Theorem 5 ([15]). Let $G$ be a graph. Then, the following conditions are equivalent:

1. $G$ is triangle-free, and $I(G)$ is Gorenstein.

2. $S / I(G)^{2}$ is Cohen-Macaulay.

\section{The Second Power of Edge Ideals}

In this section, we show that the Cohen-Macaulay and $\left(\mathrm{S}_{2}\right)$ properties are equivalent for the second power of an edge ideal.

Lemma 1. Let $G$ be a graph with $\alpha(G) \geq 2$. The following conditions are equivalent:

1. $S / I(G)^{(2)}$ satisfies the $\left(S_{2}\right)$ property,

2. $G$ is a well-covered graph and satisfies diam $\Delta\left(G_{F}\right) \leq 2$ for all the independent sets $F$ of $G$ such that $|F| \leq \alpha(G)-2$,

3. $G_{a b}$ is well-covered and satisfies $\alpha\left(G_{a b}\right)=\alpha(G)-1$ for all $a b \in E(G)$.

Proof. $(1) \Leftrightarrow(2)$ : By [12], Theorem 8.3, $I(G)$ satisfies the $\left(S_{2}\right)$ property if so does $S / I(G)^{(2)}$. Using [12], Corollary 5.4, we obtain that $\Delta(G)$ is pure. This means that $G$ is well-covered, and thus:

$$
\operatorname{dim}_{\operatorname{link}}^{\Delta(G)}(F)=\operatorname{dim} \Delta(G)-|F|
$$

and link $_{\Delta(G)}(F)=\Delta\left(G_{F}\right)$. The result is implied by Theorem 3 .

(2) $\Rightarrow$ (3): For all $a b \in E(G)$, we have:

$$
\alpha\left(G_{a b}\right) \leq \alpha(G)-1
$$

Let $F$ be an independent set of $G_{a b}$. If $|F|<\alpha(G)-1$, then $|F| \leq \alpha(G)-2$. Recall that $G_{a b}=G \backslash\left(N_{G}(a) \cup N_{G}(b)\right)$ and $F \subseteq V\left(G_{a b}\right)$. This implies that $a, b \notin N_{G}[F]$. Hence, we obtain that $\{a, b\}$ is an edge of $G_{F}$. In other words, $\{a, b\}$ is not an independent set of $G_{F}$. By the assumption, $\operatorname{diam} \Delta\left(G_{F}\right) \leq 2$, there is a vertex $c \in V\left(G_{F}\right)$ such that $\{a, c\},\{c, b\}$ are independent sets of $G_{F}$. Thus, $a c, b c \notin E\left(G_{F}\right)$. Hence, $c \in V\left(G_{a b}\right)$. Therefore, $F \cup\{c\}$ is an independent of $G_{a b}$. Then, $G_{a b}$ is well-covered, and moreover, $\alpha\left(G_{a b}\right)=\alpha(G)-1$.

(3) $\Rightarrow(2)$ : By [15], Lemma 4.1 (2), $G$ is a well-covered graph. We will prove that $\operatorname{diam} \Delta\left(G_{F}\right) \leq 2$ for all independent set $F$ with $|F| \leq \alpha(G)-2$ by induction on $\alpha(G)$.

If $\alpha(G)=2$, then we must prove diam $\Delta(G) \leq 2$. For all $a, b \in V(G)$, we assume $\{a, b\} \notin \Delta(G)$. Then, $a b \in E(G)$. By the assumption, $\alpha\left(G_{a b}\right)=\alpha(G)-1=1>0$. Therefore, we can take a vertex $c$ in $G_{a b}$, and thus, $a c, b c \notin E(G)$. Hence, $\{a, c\},\{b, c\} \in \Delta(G)$. Therefore, we conclude that diam $\Delta(G) \leq 2$.

Let $\alpha(G)>2$, and suppose that the assertion is true for all graphs $G^{\prime}$ with the same structure as $G$ satisfying the condition " $G_{a b}$ is well-covered and satisfies $\alpha\left(G_{a b}\right)=\alpha(G)-1$ for all ab $\in E(G)$ " with $\alpha\left(G^{\prime}\right)<\alpha(G)$. For all independent set $F$ of $G$ such that $|F| \leq \alpha(G)-2$, we divide the proof into the following two cases:

Case 1: $F=\varnothing$. In this case, we need to prove that diam $\Delta(G) \leq 2$. In fact, using the same argument as above, we obtain $\operatorname{diam} \Delta(G) \leq 2$.

Case 2: $F \neq \varnothing$. Let $x \in F$. Recall that $G$ is a well-covered graph, and thus, we have $\alpha\left(G_{x}\right)=\alpha(G)-1$. Hence, $|F \backslash\{x\}|=|F|-1 \leq \alpha(G)-3=\alpha\left(G_{x}\right)-2$. Note that for all $a b \in E\left(G_{x}\right)$, we have that $\left(G_{x}\right)_{a b}$ and $\left(G_{a b}\right)_{x}$ are two induced subgraphs of $G$ on vertex set $V(G) \backslash\left(N_{G}[x] \cup N_{G}(a) \cup\right.$ $\left.N_{G}(b)\right)$. Thus, $\left(G_{x}\right)_{a b}=\left(G_{a b}\right)_{x}$. By the assumption and [15], Lemma $4.1(1),\left(G_{a b}\right)_{x}$ is a well-covered graph with $\alpha\left(\left(G_{a b}\right)_{x}\right)=\alpha\left(G_{a b}\right)-1$. Therefore, $\left(G_{x}\right)_{a b}$ is also a well-covered graph. Moreover,

$$
\alpha\left(\left(G_{x}\right)_{a b}\right)=\alpha\left(\left(G_{a b}\right)_{x}\right)=\alpha\left(G_{a b}\right)-1=\alpha(G)-2=\alpha\left(G_{x}\right)-1 .
$$


Thus, $G_{x}$ has the same structure as $G$ satisfying the condition " $G_{a b}$ is well-covered and satisfies $\alpha\left(G_{a b}\right)=\alpha(G)-1$ for all $a b \in E(G)$ " with $\alpha\left(G_{x}\right)<\alpha(G)$. By the induction hypothesis, we obtain $\operatorname{diam} \Delta\left(\left(G_{x}\right)_{F \backslash\{x\}}\right) \leq 2$. Note that:

$$
\left(G_{x}\right)_{F \backslash\{x\}}=G_{x} \backslash N_{G}[F \backslash\{x\}]=G \backslash\left(N_{G}[x] \cup N_{G}[F \backslash\{x\}]\right)=G \backslash\left(N_{G}[F]\right)=G_{F} .
$$

Therefore, $\Delta\left(G_{F}\right)=\Delta\left(\left(G_{x}\right)_{F \backslash\{x\}}\right)$. Therefore, we conclude that diam $\Delta\left(G_{F}\right) \leq 2$.

Then, we get the following theorem.

Theorem 6. Let $G$ be a graph. The following conditions are equivalent:

1. $S / I(G)^{2}$ satisfies the $\left(S_{2}\right)$ property,

2. $S / I(G)^{2}$ is Cohen-Macaulay,

3. $G$ is triangle-free, and $G_{a b}$ is a well-covered graph with $\alpha\left(G_{a b}\right)=\alpha(G)-1$ for all ab $\in E(G)$.

Proof. By the statements of Conditions (1), (2) and (3), without loss of generality, we can assume that $G$ contains no isolated vertices.

$(2) \Leftrightarrow(3)$ : By [15], Theorem 4.4, $S / I(G)^{2}$ is Cohen-Macaulay if and only if $G$ is triangle-free and in $W_{2}$, which is a well-covered graph such that the removal of any vertex of $G$ leaves a well-covered graph with the same independence number as G. By [15], Lemma 4.2, this is equivalent to the condition that $G$ is triangle-free and $G_{a b}$ is a well-covered graph with $\alpha\left(G_{a b}\right)=\alpha(G)-1$ for all $a b \in E(G)$.

$(2) \Rightarrow(1)$ : It is obvious.

$(1) \Rightarrow(3)$ : If $\alpha(G)=1$, then $G$ is a complete graph. By the assumption, $G$ is one edge. Therefore, the statement holds true. Now, we assume $\alpha(G) \geq 2$. We know that $S / I(G)^{2}$ satisfies that $\left(S_{2}\right)$ property if and only if $S / I(G)^{(2)}$ satisfies the $\left(S_{2}\right)$ property and $I(G)^{2}$ has no embedded associated prime, which means $I(G)^{2}=I(G)^{(2)}$. By Theorem 4 and Lemma 1, $G$ is triangle-free, and $G_{a b}$ is well-covered with $\alpha\left(G_{a b}\right)=\alpha(G)-1$ for all $a b \in E(G)$.

Question. If $S / I(G)^{(2)}$ satisfies the $\left(S_{2}\right)$ property, then is it Cohen-Macaulay?

The question is affirmative if $G$ is a triangle-free graph by Theorems 4 and 6.

\section{Classification}

The purpose of the section is to classify all graphs $G$ such that $S / I(G)^{2}$ is Cohen-Macaulay with dimension less than five. First, we give an upper bound of the number of vertices of a graph $G$ such that $S / I(G)^{2}$ is Cohen-Macaulay.

\subsection{Upper Bound of the Number of Vertices}

Theorem 7 (Upper bound). Let $G$ be a graph with the vertex set $[n]$. Suppose $G$ has no isolate vertex. If $S / I(G)^{2}$ is d-dimensional Cohen-Macaulay, where $d \geq 3$, then we have $n \leq \frac{d^{2}+3 d-2}{2}$.

Proof. We prove this by induction on $d$. For $d=3$, we have $n \leq 8$ by [5] (see Proposition 3). Set $N(d)=\frac{d^{2}+3 d-2}{2}$. Let $n$ be the number of vertices of $G$ such that $S / I(G)^{2}$ is $d$-dimensional and Cohen-Macaulay. Let $i \in[n]$. Then, we have $n=\left|V\left(\operatorname{star}_{\Delta(G)}\{i\}\right)\right|+\mid\left([n] \backslash V\left(\operatorname{star}_{\Delta(G)}\{i\}\right) \mid\right.$. Since $G$ is triangle-free by Theorem 5, an edge among $\{i, p\},\{i, q\}$ and $\{p, q\}$ belongs to $\Delta(G)$ for any $p, q \in\left([n] \backslash V\left(\operatorname{star}_{\Delta(G)}\{i\}\right)\right.$, where $p \neq q$. By the definition of $\operatorname{star}_{\Delta(G)}\{i\}$, we have $\{i, p\},\{i, q\} \notin \Delta(G)$. Then, we have $\{p, q\} \in \Delta(G)$. By the fact that $I(G)$ is generated in degree two, all minimal non-faces of $\Delta(G)$ have cardinality two. Now, we know that $\{p, q\} \in \Delta(G)$ for any $p, q \in\left([n] \backslash V\left(\operatorname{star}_{\Delta(G)}\{i\}\right)\right.$; hence, we have $[n] \backslash V\left(\operatorname{star}_{\Delta(G)}\{i\}\right) \in \Delta(G)$. By the assumption that $S / I(G)^{2}$ is $d$-dimensional, we have $\left|[n] \backslash V\left(\operatorname{star}_{\Delta(G)}\{i\}\right)\right| \leq d$. Since $\Delta(G)$ is Gorenstein*, so is $\operatorname{link}_{\Delta(G)}\{i\}$ by [10], Theorem 
5.1. By Theorem 5, $I_{\operatorname{link}_{\Delta(G)}}^{2}\{i\}$ is Cohen-Macaulay. Hence, $\left|V\left(\operatorname{star}_{\Delta(G)}\{i\}\right)\right|=\left|V\left(\operatorname{link}_{\Delta(G)}\{i\}\right)\right|+1 \leq$ $N(d-1)+1$ by the induction hypothesis. Therefore, $n \leq N(d-1)+d+1=\frac{(d-1)^{2}+3(d-1)-2}{2}+d+1=$ $\frac{d^{2}+3 d-2}{2}=N(d)$.

\subsection{Classification}

In this subsection, we classify all graphs $G$ such that $S / I(G)^{2}$ is Cohen-Macaulay with dimension less than five.

Proposition 1. (One-dimensional case) Let $G$ be a graph with the vertex set $[n]$. Suppose $G$ has no isolate vertex. Then, $S / I(G)^{2}$ is one-dimensional Cohen-Macaulay if and only if $n=2$ and $I(G)=\left(x_{1} x_{2}\right)$.

Proposition 2 ([4]). (Two-dimensional case) Let $G$ be a graph with the vertex set $[n]$. Suppose $G$ has no isolate vertex. Then, $S / I(G)^{2}$ is two-dimensional Cohen-Macaulay if and only if $I(G)$ is one of the following up to the permutation of variables:

1. If $n=4$, then $\left(x_{1} x_{3}, x_{2} x_{4}\right)$.

2. If $n=5$, then $\left(x_{1} x_{3}, x_{1} x_{4}, x_{2} x_{3}, x_{2} x_{5}, x_{4} x_{5}\right)$.

Proposition 3 ([5]). (Three-dimensional case) Let $G$ be a graph with the vertex set $[n]$. Suppose $G$ has no isolate vertex. Then, $S / I(G)^{2}$ is three-dimensional Cohen-Macaulay if and only if $I(G)$ is one of the following up to the permutation of variables:

1. If $n=6$, then $\left(x_{1} x_{4}, x_{2} x_{5}, x_{3} x_{6}\right)$.

2. If $n=7$, then $\left(x_{1} x_{5}, x_{1} x_{6}, x_{2} x_{5}, x_{2} x_{7}, x_{3} x_{4}, x_{6} x_{7}\right)$.

3. If $n=8$, then $\left(x_{1} x_{2}, x_{1} x_{5}, x_{1} x_{8}, x_{2} x_{3}, x_{3} x_{4}, x_{4} x_{5}, x_{4} x_{8}, x_{5} x_{6}, x_{6} x_{7}, x_{7} x_{8}\right)$.

Using a computer with Nauty [16] and CoCoA [17], we classify four-dimensional case: By Theorem 7, it is enough to search for them up to $n=13$.

Theorem 8. (Four-dimensional case) Let $G$ be a graph with the vertex set $[n]$. Suppose $G$ has no isolate vertex. Then, $S / I(G)^{2}$ is four-dimensional Cohen-Macaulay if and only if $I(G)$ is one of the following up to the permutation of variables:

1. If $n=8$, then $\left(x_{1} x_{5}, x_{2} x_{6}, x_{3} x_{7}, x_{4} x_{8}\right)$.

2. If $n=9$, then $\left(x_{1} x_{5}, x_{2} x_{6}, x_{3} x_{7}, x_{1} x_{8}, x_{4} x_{8}, x_{4} x_{9}, x_{5} x_{9}\right)$.

3. If $n=10$, then

(a) $\left(x_{1} x_{5}, x_{2} x_{6}, x_{3} x_{7}, x_{1} x_{8}, x_{4} x_{8}, x_{2} x_{9}, x_{4} x_{9}, x_{5} x_{9}, x_{4} x_{10}, x_{5} x_{10}, x_{6} x_{10}\right)$.

(b) $\quad\left(x_{1} x_{5}, x_{2} x_{6}, x_{1} x_{7}, x_{3} x_{7}, x_{3} x_{8}, x_{5} x_{8}, x_{2} x_{9}, x_{4} x_{9}, x_{4} x_{10}, x_{6} x_{10}\right)$.

4. If $n=11$, then

(a) $\left(x_{1} x_{5}, x_{2} x_{6}, x_{3} x_{7}, x_{1} x_{8}, x_{4} x_{8}, x_{2} x_{9}, x_{4} x_{9}, x_{5} x_{9}, x_{3} x_{10}, x_{4} x_{10}, x_{5} x_{10}, x_{6} x_{10}, x_{4} x_{11}, x_{5} x_{11}, x_{6} x_{11}, x_{7} x_{11}\right)$.

(b) $\left(x_{1} x_{5}, x_{2} x_{6}, x_{1} x_{7}, x_{3} x_{7}, x_{3} x_{8}, x_{5} x_{8}, x_{2} x_{9}, x_{4} x_{9}, x_{1} x_{10}, x_{4} x_{10}, x_{6} x_{10}, x_{4} x_{11}, x_{5} x_{11}, x_{6} x_{11}, x_{7} x_{11}\right)$.

5. If $n=12$, then

$$
\begin{aligned}
& \left(x_{1} x_{5}, x_{2} x_{6}, x_{1} x_{7}, x_{3} x_{7}, x_{2} x_{8}, x_{4} x_{8}, x_{2} x_{9}, x_{3} x_{9}, x_{5} x_{9}, x_{1} x_{10}, x_{4} x_{10}, x_{6} x_{10}, x_{4} x_{11}, x_{5} x_{11}, x_{6} x_{11},\right. \\
& \left.x_{7} x_{11}, x_{3} x_{12}, x_{5} x_{12}, x_{6} x_{12}, x_{8} x_{12}\right) .
\end{aligned}
$$

6. If $n=13$, then

$$
\begin{aligned}
& \left(x_{1} x_{5}, x_{2} x_{6}, x_{1} x_{7}, x_{3} x_{7}, x_{2} x_{8}, x_{4} x_{8}, x_{2} x_{9}, x_{3} x_{9}, x_{5} x_{9}, x_{1} x_{10}, x_{3} x_{10}, x_{4} x_{10}, x_{6} x_{10}, x_{3} x_{11}, x_{5} x_{11}, x_{6} x_{11},\right. \\
& \left.x_{8} x_{11}, x_{2} x_{12}, x_{4} x_{12}, x_{5} x_{12}, x_{7} x_{12}, x_{4} x_{13}, x_{6} x_{13}, x_{7} x_{13}, x_{9} x_{13}\right) .
\end{aligned}
$$


See [18] for the concrete algorithm we used. By Theorem 6 in this case, the Cohen-Macaulay property is equivalent to the $\left(S_{2}\right)$ property, which is independent of the base field $K$.

\section{Example}

In this section, we give an example of a Gorenstein squarefree monomial ideal $I$ such that $S / I^{2}$ satisfies the Serre condition $\left(\mathrm{S}_{2}\right)$, but it is not Cohen-Macaulay.

The Cohen-Macaulay property of $I_{\Delta}^{2}$ implies the "Gorenstein" property of $I_{\Delta}$. More precisely:

Theorem 9 ([7]). Let $\Delta$ be a simplicial complex on $[n]$. Suppose that $S / I_{\Delta}^{2}$ is Cohen-Macaulay over any field $K$. Then, $\Delta$ is Gorenstein for any field $K$.

In [7], the authors asked the following question:

Question. Let $\Delta$ be a simplicial complex on $[n]$. Let $S=K\left[x_{1}, \ldots, x_{n}\right]$ be a polynomial ring for a fixed field $K$. Suppose $\Delta$ satisfies the following conditions:

1. $\Delta$ is Gorenstein.

2. $S / I_{\Delta}^{2}$ satisfies the Serre condition $\left(S_{2}\right)$.

Then, is it true that $S / I_{\Delta}^{2}$ is Cohen-Macaulay?

Using a list in [19] and CoCoA, we have the following counter-example:

Example 1. Let $K$ be a field of characteristic zero. Set:

$I_{\Delta}=\left(x_{1} x_{10}, x_{3} x_{9}, x_{2} x_{9}, x_{7} x_{8}, x_{2} x_{8}, x_{4} x_{7}, x_{5} x_{6}, x_{3} x_{6}, x_{4} x_{5}, x_{6} x_{8} x_{10}, x_{2} x_{5} x_{10}, x_{1} x_{4} x_{9}, x_{1} x_{3} x_{7}\right)$.

Then, the following conditions hold:

1. $\Delta$ is Gorenstein.

2. $S / I_{\Delta}^{2}$ satisfies the Serre condition $\left(S_{2}\right)$.

3. $S / I_{\Delta}^{2}$ is not Cohen-Macaulay.

We explain how to find the example. The manifold page of Lutz [19] gives a classification of all triangulations $\Delta$ of the three-sphere with 10 vertices, which shows that there are 247,882 types. Using Theorem 3, we checked the Serre condition $\left(S_{2}\right)$ for them, and there were only nine types such that $S / I_{\Delta}^{2}$ satisfies the Serre condition $\left(S_{2}\right)$. Among the nine types, there was only one simplicial complex $\Delta$ such that $S / I_{\Delta}^{2}$ is not Cohen-Macaulay, which is the above example. Note that a triangulation $\Delta$ of a sphere is always Gorenstein. See [18] for more information.

Author Contributions: These authors contributed equally to this work.

Funding: This work was partially supported by a JSPS Grant-in Aid for Scientific Research (C) 18 K03244. The research of Do Trong Hoang was supported by the project ICRTM01 2019.02 of the International Center for Research and Postgraduate Training in Mathematics, VAST.

Conflicts of Interest: The authors declare no conflict of interest.

\section{References}

1. Crupi, M.; Rinaldo, G.; Terai, N. Cohen-Macaulay edge ideal whose height is half of the number of vertices. Nagoya Math. J. 2011, 201, 117-131. [CrossRef]

2. Terai, N.; Yoshida, K. A note on Cohen-Macaulayness of Stanley-Reisner rings with Serre's condition $\left(\mathrm{S}_{2}\right)$. Comm. Algebra 2008, 36, 464-477. [CrossRef] 
3. Terai, N.; Trung, N.V. Cohen-Macaulayness of large powers of Stanley-Reisner ideals. Adv. Math. 2012, 229, $711-730$. [CrossRef]

4. Minh, N.C.; Trung, N.V. Cohen-Macaulayness of powers of two-dimensional squarefree monomial ideals. J. Algebra 2009, 322, $4219-4227$. [CrossRef]

5. Trung, N.V.; Tuan, T.M. Equality of ordinary and symbolic powers of Stanley-Reisner ideals. J. Algebra 2011, 328, 77-93. [CrossRef]

6. Hoang, D.T.; Minh, N.C.; Trung, T.N. Cohen-Macaulay graphs with large girth. J. Algebra Appl. 2015, 14, 1550112. [CrossRef]

7. Rinaldo, G.; Terai, N.; Yoshida, K. On the second powers of Stanley-Reisner ideals. J. Commut. Algebra 2011, 3, 405-430. [CrossRef]

8. Crupi, M.; Rinaldo, G.; Terai, N.; Yoshida, K. Effective Cowsik-Nori theorem for edge ideals. Comm. Algebra 2010, 38, 3347-3357. [CrossRef]

9. Bruns, W.; Herzog, J. Cohen-Macaulay Rings; Cambridge Univ. Press: Cambridge, UK, 1993.

10. Stanley, R.P. Combinatorics and Commutative Algebra, 2nd ed.; Birkhäuser: Boston, MA, USA; Basel, Switzerland; Stuttgart, Germany, 1996.

11. Villarreal, R.H. Monomial Algebra, 2nd ed.; CRC Press: Boca Raton, FL, USA; London, UK; New York, NY, USA, 2015.

12. Pournaki, M.R.; Seyed Fakhari, S.A.; Terai, N.; Yassemi, S. Survey article: Simplicial complexes satisfying Serre's condition: a survey with some new results. J. Commut. Algebra 2014, 6, 455-483. [CrossRef]

13. Rinaldo, G.; Terai, N.; Yoshida, K. Cohen-Macaulayness for symbolic power ideals of edge ideals. J. Algebra 2011, 347, 1-22. [CrossRef]

14. Simis, A.; Vasconcelos, W.V.; Villarreal, R.H. On the ideal theory of graphs. J. Algebra 1994, 167, $389-416$. [CrossRef]

15. Hoang, D.T.; Trung, T.N. A characterization of triangle-free Gorenstein graphs and Cohen-Macaulayness of second powers of edge ideals. J. Algebr. Combin. 2016, 43, 325-338. [CrossRef]

16. McKay, B.D. NAUTY: No AUTomorphisms, Yes? Available online: http://cs.anu.edu.au/\{ \}bdm/nauty/ (accessed on 29 June 2019).

17. Abbott, J.; Bigatti, A.M.; Robbiano, L. CoCoA: A system for doing Computations in Commutative Algebra. Available online: http:/ / cocoa.dima.unige.it (accessed on 29 June 2019).

18. Hoang, D.T.; Rinaldo, G.; Terai, N. Cohen-Macaulay and S2 of Second Power. Available online: http: / / www.giancarlorinaldo.it/cm-s2-2nd-power.html (accessed on 29 June 2019).

19. Lutz, F.H. The Manifold Page. Available online: http://page.math.tu-berlin.de/\$ sim\$lutz/stellar/ (accessed on 29 June 2019).

(C) 2019 by the authors. Licensee MDPI, Basel, Switzerland. This article is an open access article distributed under the terms and conditions of the Creative Commons Attribution (CC BY) license (http://creativecommons.org/licenses/by/4.0/). 